\title{
Long- and short-time analysis of heartbeat sequences: Correlation with mortality risk in congestive heart failure patients
}

\author{
P. Allegrini,${ }^{1}$ R. Balocchi, ${ }^{2}$ S. Chillemi, ${ }^{3}$ P. Grigolini,${ }^{3,4,5}$ P. Hamilton, ${ }^{6}$ R. Maestri, ${ }^{7}$ L. Palatella, ${ }^{5}$ and G. Raffaelli ${ }^{8}$ \\ ${ }^{1}$ Istituto di Linguistica Computazionale del Consiglio Nazionale delle Ricerche, Area della Ricerca di Pisa-S. Cataldo, Via Moruzzi 1, \\ 56124, Ghezzano-Pisa, Italy \\ ${ }^{2}$ Istituto di Fisiologia Clinica del Consiglio Nazionale delle Ricerche, Area della Ricerca di Pisa-S. Cataldo, Via Moruzzi 1, \\ 56124, Ghezzano-Pisa, Italy \\ ${ }^{3}$ Istituto di Biofisica del Consiglio Nazionale delle Ricerche, Area della Ricerca di Pisa-S. Cataldo, Via Moruzzi 1, \\ 56124, Ghezzano-Pisa, Italy \\ ${ }^{4}$ Center for Nonlinear Science, University of North Texas, P.O. Box 311427, Denton, Texas, 76203-1427, USA \\ ${ }^{5}$ Dipartimento di Fisica dell'Università di Pisa and INFM, Via Buonarroti 2, 56127 Pisa, Italy \\ ${ }^{6}$ Center for Nonlinear Science, Texas Woman's University, P.O. Box 425498, Denton, Texas 76204, USA \\ ${ }^{7}$ Fondazione S. Maugeri, IRCCS, Istituto Scientifico di Montescano, Via per Montescano, 27040 Montescano (PV), Italy \\ ${ }^{8}$ International School for Advanced Studies and INFM Unit, Via Beirut 2-4, 34014 Trieste, Italy \\ (Received 23 July 2002; revised manuscript received 14 February 2003; published 23 June 2003)
}

\begin{abstract}
We analyze $R R$ heartbeat sequences with a dynamic model that satisfactorily reproduces both the long- and the short-time statistical properties of heart beating. These properties are expressed quantitatively by means of two significant parameters, the scaling $\delta$ concerning the asymptotic effects of long-range correlation, and the quantity $1-\pi$ establishing the amount of uncorrelated fluctuations. We find a correlation between the position in the phase space $(\delta, \pi)$ of patients with congestive heart failure and their mortality risk.
\end{abstract}

DOI: 10.1103/PhysRevE.67.062901

PACS number(s): 87.19.Hh, 02.50.Ey, 05.20.-y, 05.40.Fb

The main purpose of this Brief Report is twofold. The first goal is to afford a firmer theoretical basis to the method of analysis of heartbeating proposed in an earlier publication [1]. The second goal is to substantiate with more convincing arguments that this method yields an efficient criterion to estimate the mortality risk of congestive heart failure (CHF) patients.

To prove this significant diagnostic property, we illustrate the relevant clinical data, missing in the earlier publication, and we limit ourselves to affording only this kind of "experimental" information. For other details, already adequately illustrated in the earlier publication, we refer the interested reader to Ref. [1]. For this study we considered 13 male CHF patients, from a study base of 320 subjects, who experienced cardiac death during a follow-up of 26 months (average 19 months, median 22 months). Inclusion criteria were absence of pulmonary or neurological disease, absence of acute myocardial infarction or cardiac surgery within the previous six months, absence of any other disease limiting survival, stable therapy for at least two weeks, and good quality 24-h Holter recordings, with an ectopy rate less than 5\%. A comparable number of control subjects (16 patients), matching for age, sex, NYHA class (a functional and therapeutic classification for prescription of physical activity for cardiac patients), and etiology, was then selected. These latter patients did not experience cardiac death after follow-up. All patients had a 24-h Holter recording at baseline, together with standard functional evaluation including measurement of left ventricular ejection fraction, peak VO2 $\left(\mathrm{O}_{2}\right.$ consumption during effort), and sodium (Na). Finally, $R R$ series for 10 healthy subjects were taken from the Nonlinear Time Series Analysis (NOLTISALIS) archive [2].

The signal that we plan to analyze is the sequence $\left\{T_{i}\right\}$, illustrated by Fig. 2 of Ref. [1]. Actually, this signal is de- rived from the true experimental signal, the electrocardiogram (ECG), by measuring the distance between two nearest neighbor $R$ pulses. Thus a given value $T_{i}$ of the sequence to analyze is the time distance between the $i$ th and the ( $i$ $+1)$ th pulse. It is called an $R R$ sequence. The sequence $\left\{T_{i}\right\}$ can be studied as a new time series, with $i$ playing the role of "time." Moreover, the value $T_{i}$, expressed as a function of $i$ with $i \gg 1$, can be thought of as a function $T(t)$, namely, as a function of a continuous time variable $t$.

The second step of the method of Ref. [1], as shown here by Fig. 1 , is based on dividing the plane $(T, t)$ into horizontal strips of size $s$ and on recording the lengths of the sojourn times of $T(t)$ in a strip. This resulted in a waiting time distribution $\psi(t)$ that did not agree with the scaling value ob-

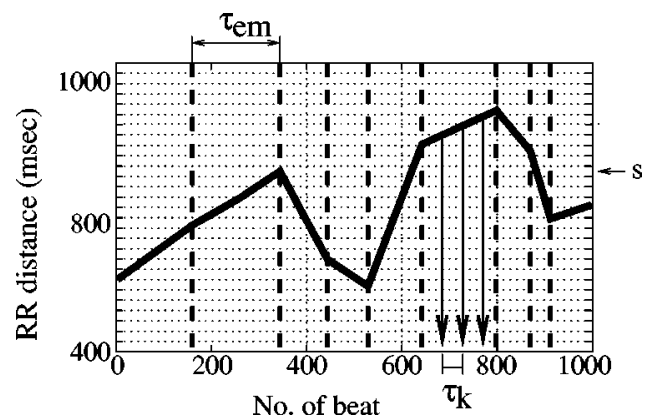

FIG. 1. A sketch of the EM model. The dashed vertical lines delimit the zones of constant slope, of length $\tau_{e m}$, one of which (the one that contained the 200th beat) is explicitly indicated by the double arrow. The horizontal stripes signaled by dotted lines delimit the path that the trajectory $T(t)$ should follow to remain within the same cell. The vertical arrows signal the times of transition from one to another cell, in the case of the laminar region between 600 and 800 beats. 
served numerically by using the method of scaling detection of Refs. $[3,4]$.

We now prove that the numerical results of Ref. [1] can be accounted for very well by means of a model that we call the extended memory (EM) model. The real signal $T(t)$ is obtained by adding uncorrelated noise: this gives the impression that $T(t)$ is totally erratic, while it is not. Let us describe the EM model. First of all, we assume that for a given time $\tau_{e m}$, the curve $T(t)$ retains a given slope $\alpha$, then it abruptly gets a new slope $\alpha^{\prime}$, for an interval of time $\tau_{e m}^{\prime}$, after which a new abrupt transition to a new slope $\alpha^{\prime \prime}$ takes place, for a time $\tau_{e m}^{\prime \prime}$, and so on. It is evident that the resulting $T(t)$ has the form of the zigzag curve illustrated in Fig. 1 [5]. We shall refer to the individual straight line intervals of this curve as laminar regions. Any laminar region is associated with its own $\tau_{e m}$. We assign to the waiting time distribution $\psi\left(\tau_{e m}\right)$ the inverse power law form

$$
\psi\left(\tau_{e m}\right)=(\nu-1) \frac{\left[\left\langle\tau_{e m}\right\rangle(\nu-2)\right]^{\nu-1}}{\left[\left\langle\tau_{e m}\right\rangle(\nu-2)+\tau_{e m}\right]^{\nu}},
$$

with $2<\nu<3$, where $\left\langle\tau_{e m}\right\rangle$ is the average waiting time.

We have to discuss the EM model in the light of the analysis that will be applied to a real signal, where the laminar regions are not directly observable. To perform the analysis we define a coarse-graining parameter $s$ and we obtain a new series, namely,

$$
N_{i}^{(s)} \equiv\left[T_{i} / s\right],
$$

where $N_{i}^{(s)}$ is the coarse-grained time series and [a] denotes the integer part of $a$. To help the reader to understand how the new sequence is created, we note that the prescription of Eq. (2) is equivalent to dividing the $R R$ axis into cells of equal size $s$. The sequence $T_{i}$ is replaced by integer numbers with the same value if the trajectory $T(t)$ remains in the same cell. Next, to make our approach as fast and accurate as possible, we adopt the walking prescription of Ref. [4] by setting the rule $\xi_{i}=1$ when $N_{i}^{(s)} \neq N_{i-1}^{(s)}$, and $\xi_{i}=0$ if $N_{i}^{(s)}$ $=N_{i-1}^{(s)}$. Finally, we generate several trajectories (labeled with the index $l$ ) for the variable $x_{l}$ at "time" $t$, namely,

$$
x_{l}(t)=\sum_{i=l}^{l+t} \xi_{i} .
$$

Note that, for simplicity, we have omitted indicating the dependence on $s$.

With the help of Fig. 1 we show that the coarse-graining procedure results in significant correlation, or, equivalently, in many pseudoevents. The double arrow with the label $\tau_{e m}$ indicates a typical laminar region of length $\tau_{e m}$. The vertical arrows denote the locations of 1's. For any laminar region with slope $\alpha$, there are approximately $\tau_{e m}|\alpha| / s$ time intervals of sojourn within a cell, of duration $\tau_{k}=s /|\alpha|$. In other words, the experimental observation yields a distribution of times $\tau_{k}$. This is determined by the distribution of Eq. (1) but it does not coincide with it. The larger either $\tau_{e m}$ or $|\alpha|$, the larger is the number of correlated events. As earlier anticipated, to simulate the real signal, we add to the zigzag curve a totally random fluctuation. The numbers of shorttime intervals, and their lenghts $\tau_{k}$ as well, are significantly modified by this additional random fluctuation.

Now, we want to explain why the adoption of the method of statistical analysis, used in Ref. [1] and applied again in this Brief Report, reveals the existence of the real scaling, in spite of the arbitrary coarse-graining method adopted. The technique of analysis adopted is that originally proposed in Refs. [3,4]. It rests on using the trajectories of Eq. (3) to create a diffusion process, and we determine numerically the probability distribution of $x$ at time $t$, denoted by $p(x, t)$. Then, we evaluate the Shannon entropy [6] of this diffusion process. This is the reason why this method of analysis is called the diffusion entropy (DE) method. As shown in Ref. [4], if the sequence $\left\{\xi_{i}\right\}$ is ergodic, with mean value $\bar{\xi}$, then $p(x, t)$ is expected to fit the scaling property

$$
p(x-\bar{\xi} t, t)=\frac{1}{t^{\delta}} F\left(\frac{x-\bar{\xi} t}{t^{\delta}}\right),
$$

with the departure of $\delta$ from the usual value 0.5 measuring the complexity of the process. It is straightforward to prove that the Shannon entropy

$$
S(t)=-\int_{-\infty}^{\infty} p(x, t) \ln [p(x, t)] d x
$$

of a process fitting the scaling condition of Eq. (4) yields

$$
S(t)=A+\delta \ln (t)
$$

where $A$ is a constant, whose explicit form is not relevant for the ensuing discussion.

We now make the assumption that the times $\tau_{e m}$ of Eq. (1) are uncorrelated. We shall prove that this assumption yields

$$
\delta=\frac{1}{\nu-1} .
$$

The DE analysis is made on sequences of $\tau_{k}$ values, which are $s$ dependent. However, $\nu$ is a property of the hidden EM model, thereby implying that Eq. (7) is independent of the coarse-graining. Let us consider first the ideal case where no short-time randomness is present, and define the distribution probability for the slope $\alpha, f(\alpha)$. We make the simplifying and plausible hypothesis that this distribution does not have infinite moments. Note that this restriction does not apply to the $\tau$ distribution, which has, in fact, slow tails and diverging moments. During one single trend of acceleration or deceleration the position of the walker $x_{l}(t)$ is displaced by a quantity

$$
z=\frac{|\alpha|}{s} \tau_{e m},
$$


which is the number of times the walker crosses the "box boundaries" defined by the coarse-graining procedure on $\left\{T_{i}\right\}$. Consequently, we can write the probability of a displacement $z$ as

$$
p(z)=\int_{0}^{\infty} d \tau_{e m} \int_{-\infty}^{\infty} d \alpha \delta\left(z-\frac{|\alpha| \tau_{e m}}{s}\right) \psi\left(\tau_{e m}\right) f(\alpha) .
$$

In the long-time limit [7] this process becomes indistinguishable from a process where a walker, at regular time distances of duration $\left\langle\tau_{e m}\right\rangle$, walks by a quantity $\Delta x \sim z$, distributed as

$$
\Pi(\Delta x)=\frac{s}{|\alpha|\left\langle\tau_{e m}\right\rangle} \psi\left(\frac{s \Delta x}{|\alpha|}\right) .
$$

Since the function $\psi$ has a long tail, this latter process, according to the generalized central limit theorem [8], results in a Lévy flight, which is a stable process with diverging central moments. The asymptotic scaling property of the walker $x_{l}(t)$ is determined by the first diverging fractional moment of the distribution $p(z)$,

$$
\left\langle z^{\gamma}\right\rangle=\int_{0}^{\infty} p(z) z^{\gamma} d z
$$

The smallest value of $\gamma$ for which this integral diverges, called $\hat{\gamma}$, defines the scaling $\delta$ via $\delta=1 / \hat{\gamma}[8]$. Substituting Eq. (9) into Eq. (11), we obtain

$$
\left\langle z^{\gamma}\right\rangle=\int_{0}^{\infty} d z \int_{0}^{\infty} d \tau_{e m} \int_{-\infty}^{\infty} d \alpha \delta\left(z-\frac{\alpha \tau_{e m}}{s}\right) \psi\left(\tau_{e m}\right) f(\alpha) z^{\gamma} .
$$

Integrating over $z$, this result can be written as

$$
\left\langle z^{\gamma}\right\rangle=\left(\int_{0}^{\infty} \psi\left(\tau_{e m}\right) \tau_{e m}^{\gamma} d \tau_{e m}\right) F_{s},
$$

where

$$
F_{s}=\int_{-\infty}^{\infty} d \alpha\left(\frac{|\alpha|}{s}\right)^{\gamma} f(\alpha)
$$

This means that only the first factor might make $\left\langle\tau_{e m}^{\gamma}\right\rangle$ divergent, thereby implying that $\hat{\gamma}=\nu-1$ and proving Eq. (7). This also proves that $\delta$ does not depend either on the coarsegraining parameter $s$ or on the distribution of $\alpha$. This theoretical prediction is supported by the results of the numerical analysis, not shown here.

The value of $\delta$ does not depend on the strength of the additive noise perturbing the EM model behavior, either. In fact, the sequence $\left\{\tau_{\text {expt }}\right\}$, detected on the real $R R$ sequences, can be modeled by

$$
\tau_{\text {expt }}(t)=a \tau_{\text {em }}(t)+b R(t),
$$

with $a \ll 1$ and $b$ close to 1 . The first contribution corresponds to the EM model, and the second term $R(t)$ is gen- erated by the short-time zero-centered random fluctuations. The EM model component yields superdiffusion, while the random component generates ordinary diffusion. In the asymptotic limit of very large values of $t$, the superdiffusion component, which is faster than the ordinary diffusion, becomes predominant, and the DE method again detects the correct scaling $\delta$ even in the case where $a \ll b$. This means that the prediction of Eq. (7) holds true even when the trajectory $T(t)$, due to the action of a very strong noise, seems to deviate significantly from the behavior prescribed by the EM model.

We use Eq. (15) to get for the correlation function $C_{\text {expt }}(t)$ of the sequence $\left\{\tau_{\text {expt }}\right\}$ the expression [9-12]

$$
C_{\text {expt }}(t)=p C_{\text {em }}(t)+(1-p) C_{\text {random }}(t),
$$

where $C_{e m}(t)$ is the correlation function generated by the zigzag signal and $C_{\text {random }}(t)$ is the correlation function generated by the uncorrelated fluctuations. This means that $C_{e m}(t)$ is an inverse-power-law relaxation and $C_{\text {random }}(t)$ a relaxation function decaying to zero in one time step. With no loss of generality we assume $\left\langle\tau_{\text {em }}^{2}\right\rangle=\left\langle R^{2}\right\rangle=\left\langle\tau_{\text {expt }}^{2}\right\rangle$, implying $a^{2}+b^{2}=1$, and consequently $p=a^{2}$ and $(1-p)$ $=b^{2}$. The parameter $p$ can be evaluated by monitoring the experimental correlation function at the first time step. According to the fact that $C_{\text {random }}(t)$ decays to zero in one step, while $C_{e m}(t)$ is much slower, we immediately obtain $p$ $=C_{\text {expt }}(1)$. The parameter $p$ depends on $s$. This is so because the experimental evaluation of $\tau_{\text {expt }}(t)$ is $s$ dependent. The parameter $p$ defines the statistical weight of the EM component present in the experimental signal $T(t)$. However, its dependence on $s$ makes its use questionable. Nevertheless, numerical work, not illustrated here, shows that all curves $p(s)$ have a bell-shaped form with a clearly defined maximum, which is referred to by us as $\pi$. This maximum is a property independent of $s$. In fact, we note that almost all the healthy patients have their maximum at $30 \mathrm{~ms}$, while most of the CHF patients have theirs at $20 \mathrm{~ms}$ : the parameter $\pi$ is a reliable measure of the intensity of the EM component. Consequently, we decided to represent the conditions of all patients, healthy and CHF alike, in the $(\delta, \pi)$ plane which we call the phase plane. The criteria adopted to define the phase plane make the resulting diagram independent of the coarse-graining parameter $s$, and the location of any patient in the plane is an objective property independent of the coarse graining parameter $s$.

Figure 2 illustrates the distribution of patients in the phase space. As found in Ref. [1], the healthy patients occupy a broad region in the left top of the phase space, while the CHF patients are predominantly distributed along the diagonal connecting the right top to the left bottom of the graph, with a small overlap with the healthy region. However, the authors of Ref. [1] did not have the information necessary to prove that their approach makes it possible to estimate the mortality risk. To show this property, let us define the center of the healthy zone obtained the averaging the parameters of healthy patients. We denote this point with a white square in Fig. 2. Then, we rank the CHF patients according to their distance from the white square. In other words, the first CHF 


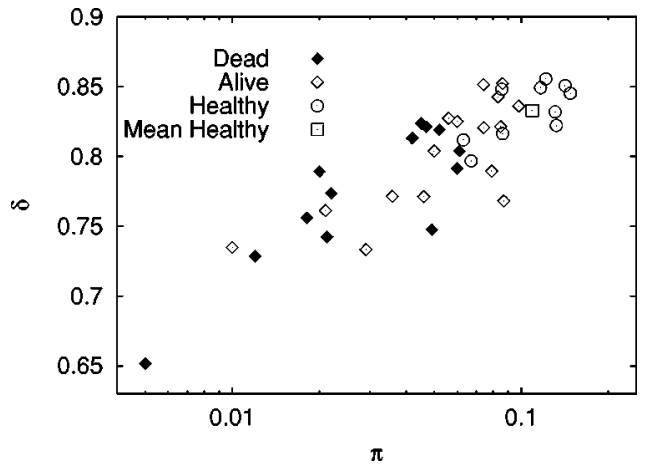

FIG. 2. Positions of healthy (circles) and CHF (diamonds) subjects in the phase plane. The white diamonds correspond to patients alive after the end of the experiment, and the black ones to patients who were either dead or urgently transplanted. The white square (optimal condition, see text) represents the average position of healthy subjects in the plot.

patient is the one with minimum distance from the optimal condition. Then we observe the remaining patients, and we rank as second the one with minimum distance from the optimal condition, and so on. We find that the first seven patients are alive. The eighth patient belongs to the group of patients who died, and from now on the patients are either alive or dead. This suggests that the closer the patient to the optimal condition the higher the survival probability. To support this important property in a more rigorous way, this important property, we used the Mann-Whitney method [13], which proved that the probability for the distribution of dead and alive patients of Fig. 2 to be fortuitous is less than $3 \%$.

Moreover, it is important to remark that the survived patients corresponding to points in the phase plane far from the optimal conditions either had a serious pathology, being classified as NYHA class III (severe physical limitations, they are comfortable only at rest) and therefore required a heart transplant anyway, or had a very short follow-up time (less than six months). It is remarkable that the six live patients who do not fit either of the earlier conditions occupy a region overlapping the zone of healthy subjects. The efficiency of this mortality risk criterion should be checked with a much larger number of patients. However, this Brief Report sets the statistical analysis behind it on a solid statistical ground.

We gratefully acknowledge financial support from the Army Research Office through Grant No. DAAD 19-020037. We also thank the S. Maugeri Foundation for supplying the data.
[1] P. Allegrini, P. Grigolini, P. Hamilton, L. Palatella, and G. Raffaelli, Phys. Rev. E 65, 041926 (2002).

[2] Available on CD-Rom: M. G. Signorini, R. Sassi, and S. Cerutti, in Proceedings of the 23th Annual Conference of the IEEE-EMBS, Istanbul, Turkey, 2001 (IEEE Press, Piscataway, NJ, 2001).

[3] N. Scafetta, P. Hamilton, and P. Grigolini, Fractals 9, 193 (2001).

[4] P. Grigolini, L. Palatella, and G. Raffaelli, Fractals 9, 439 (2001).

[5] We assume that the signal of Fig. 2 of Ref. [1] is derived from that of Fig. 1 by adding short-time noise to it.

[6] C. E. Shannon and W. Weaver, The Mathematical Theory of Communication (University of Illinois Press, Champaign, IL, 1963).

[7] M. Annunziato and P. Grigolini, Phys. Lett. A 269, 31 (2000).

[8] B. V. Gnedenko and A. N. Kolmogorov, Limit Distributions for Sums of Independent Random Variables (Addison-Wesley, Cambridge, MA, 1954).

[9] Note that in literature concerning DNA sequences there are already models yielding the result of Eq. (16). Some authors [10] refer to this kind of model as a generalized Lévy (GL) process. Other authors [11] called this kind of model a copying mistake map (CMM). In a more recent work [12] the equivalence between GL and CMM processes has been carefully discussed. We note that there is a subtle difference between the model of this Brief Report and these earlier models, due to the fact that here we have a signal that is is the sum of a long-time memory component and a short-time noise, while in the earlier models the trajectory adopted either the random or the correlated prescription, with different probabilities.

[10] S. V. Buldyrev, A. L. Goldberger, S. Havlin, C.-K. Peng, M. Simons, and H. E. Stanley, Phys. Rev. E 47, 4514 (1993).

[11] P. Allegrini, M. Barbi, P. Grigolini, and B. J. West, Phys. Rev. E 52, 5281 (1995); P. Allegrini, P. Grigolini, and B. J. West, Phys. Lett. A 211, 217 (1996).

[12] P. Allegrini, M. Buiatti, P. Grigolini, and B. J. West, Phys. Rev. E 58, 3640 (1998).

[13] M. Hollander and D. A. Wolfe, Nonparametric Statistical Methods (John Wiley and Sons, New York, 1973). 